\title{
Method of MAI cancellation in asynchronous underwater acoustic CDMA system
}

\author{
Shengjun Xiong*, Lisheng Zhou, Zhengduo Wang, Chao Wang, and Yuyan Zhang \\ Science and Technology on Sonar Laboratory, Hangzhou Applied Acoustic Research Institute, Hangzhou, P. R. China
}

\begin{abstract}
In the underwater passive positioning systems (UPPS), it involves a key technology of multiuser communication that the beacons synchronously send positioning messages to the targets. Interferences from other users, referred to multi-access interferences (MAIs), can induce significant bit errors for the desired user. In this paper, we designed an underwater acoustic Code Division Multiple Access (CDMA) system using the coded cyclic shift key (CCSK) modulation, and proposed a hybrid MAI cancellation scheme based on cyclic shift inverse matrix transform (CSIMT) and recursive least square (RLS) in the CDMA system. The hybrid scheme can suppress MAI better than RLS or CSIMT, and meets the requirements of strong stability to support reliable transmission for UPPS. The CSIMT interference cancellation performs well under the far-near effects, which doesn't depend on the accuracy of the estimation of transmitted data, and the strong MAI can be reduced by transformation of the zero-forcing correlation values using $\mathrm{m}$ sequence cyclic shift inverse matrix. The RLS interference cancellation is used to mitigate MAI form the detected users with comparable power, by using the estimated the channel impulse response between the receiver and the detected users, and the regenerated signal of detected users form decoder output, which allows of strong error-correcting codes, and it's robust in an asynchronous environment. The theoretical analysis and experimental result show that the hybrid MAI cancellation scheme can effectively suppress MAI better. The shallow water sea trial was carried out near Dalian Tiger Beach Ocean Park in China, at a distance of about $5 \mathrm{~km}$, the data rates of $100 \mathrm{bps}$, where three users could communicate asynchronously with low failure ratio and can match the UPPS application.
\end{abstract}

\section{Introduction}

Recently Underwater Acoustic Sensor Networks (UASN) have been deployed to carry out collaborative monitoring missions such as oceanographic data collection, special climate formation, disaster prevention, and biological habit change[1,2]. Regional, variable and real-time marine environmental observation requires wide coverage and fast change tracking capability of the networks, there has been increasing engineering activity in the deployment of Underwater Unmanned Vehicles (UUVs) in UASN. UUVs end owed with sensing and wireless communication capabilities become essential. Using sensors mounted on UUVs for time and space sampling of the ocean, network transmission of sensor data and positioning of UUVs have always been a concern of scholars. UUVs are often navigated by expensive inertial navigation system (INS), which gives accurate position information for short time period, while the estimated position accumulates error due to the bias error of the sensors as time passes. The more economical approach of mobile navigation support development supposes application of underwater passive positioning systems (UPPS), based on underwater acoustic communication (UAC) link[3], .In the UPPS, the GPS information from the buoys or wave gliders is sent to the UUVs by UAC at the same time, the receivers mounted on UUVs recovery information, use multiple GPS and signal arrival time to establish multiple equations, and solve the equation to obtain its own position information. The advantage of UPPS is that the high accuracy of the whole UUVs group without additional equipment.

Code Division Multiple Access (CDMA) is a suitable technique to achieve detection of arrivals and user identification in asynchronous systems. CDMA assigns a distinct sequence to each user (Direct-Sequence CDMA or DS-CDMA), which should have good autocorrelation and cross correlation properties. Thus, DS-CDMA has been used to identify different beacons in positioning systems. In the DS-CDMA, the multiple access interference (MAI) can seriously deteriorate the system's performance. In the UPPS, the users have different distances from the receiver, thus, the received signals do not have the same power at the receiver end, which is known as the near-far effects. Moreover, the arrival time of the positioning message signal at the receiver cannot be completely synchronized, which increases the difficulty of MAI suppression even further. Because nodes that need to navigate can only passively receive signals, without a closed-loop power control, the strong MAI caused by the far-near effects and asynchronous

\footnotetext{
* Corresponding author: xiongsj715@163.com
} 


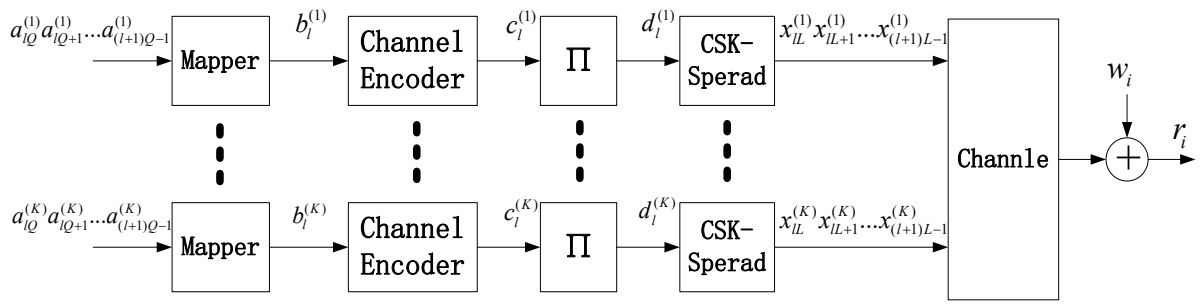

Fig. 1. A DS-CDMA system using CCSK modulation.

environment in DS-CDMA system can induce significant bit errors for the desired user.

Rake receiver was added into underwater acoustic DS-CDMA system in the early days, the performance can be improved by taking advantage of the time diversity introduced by the multi-paths[4]. However, with the increase of underwater acoustic channel dispersion, RAKE receiver's performance is limited by ISI and MAI[5], . Bit-error-ratio (BER) performance can be significantly degraded[6], if the channel estimation is not perfect. Salah A. Aliesawi proposed two kinds of CDMA multi-user detection systems and interleaved division multiple accesses with adaptive channel estimation applicable to synchronous systems, and the sea trail was conducted in 2009 for synchronous twouser scenarios[7]. From 2010 to 2013, to cope with the ISI, separation of users in the spatial domain by time reversal has been considered by S. E. Cho and $\mathrm{H}$. C. Song who investigated adaptive time reversal (ATR) with channel estimation and a vertical receive array for multi-user communications and conducted some experiments[8-11].

The literature mentioned above focus on the MAI suppression in synchronous systems with a vertical array, and can't match the UPPS application. In this paper, we propose a hybrid multi-user interference cancellation (IC) scheme based on cyclic shift inverse matrix transform (CSIMT) and recursive least square (RLS) in DS-CDMA system using the coded cyclic shift key (CCSK) modulation. We focus on the hybrid scheme due to its superior MAI suppression capability in the UPPS. The CSIMT-IC can perform well under the far-near effects, and the strong MAI can be reduced by transformation of the zero-forcing correlation values using $\mathrm{m}$ sequence cyclic shift inverse matrix. The RLS-IC is used to mitigate MAI form the detected users with comparable power, which uses the estimated channel impulse responses (CIRs) between the receiver and the detected users by the RLS algorithm. The regenerated signal of unexpected user form decoder output, allows of strong error-correcting codes, which is robust in an asynchronous environment. The hybrid scheme includes a two-stage process, firstly, the MAI of high-power users can be mitigated by using CSIMT-IC, secondly, and the MAI of users with comparable power can be mitigated by using RLS-IC. The theoretical analysis shows that the hybrid MAI cancellation scheme can effectively suppress MAI better. The shallow water sea trial was carried out near Dalian Tiger Beach Ocean Park in China, at a distance of about $5 \mathrm{~km}$, the data rates of $100 \mathrm{bps}$, where three users could communicate asynchronously with low failure ratio (the ratio of the number of failures of multi-user communication to the total number of communication. As long as the error occurs in one of the users, the communication fails).

The remainder of this article is organized as follows: The problem description and system model of DSCDMA is first reviewed in Sec.2. The CSIMT-IC method is discussed under the far-near effects in Sec.3. After that, we introduce the idea of RLS-IC method in Sec.4. A 3-user DS-CDMA system is simulated in Sec.5. In Sec.6, the sea trial multi-user data are processed by using the hybrid MAI cancellation scheme based on CSIMT and RLS. Sec. 6 gives discussion and summary.

\section{System model}

Consider a DS-CDMA system with $\mathrm{K}$ users as shown in Fig. 1. At the transmitter, $K$ bit streams, with $a_{l Q+q}^{(k)}$ being the $(l Q+q)$-th information bit of the $k$-th stream or the $k$-th user, every $Q$ coded bits with $a_{l Q}^{(k)}, a_{l Q+1}^{(k)}, \ldots, a_{(l+1) Q-1}^{(k)}$, are mapped onto the $l$-th symbol $b_{l}^{(k)}$ of the $k$-th user, where $b_{l}^{(k)}$ are independently encoded, interleaved, and the outputs of the encoder, inter-leaver, are denoted by $c_{l}^{(k)}$, and $d_{l}^{(k)}$. After interleaving, $d_{l}^{(k)}$, for $l=0,1, \cdots, N-1$, are assumed to be independent and identically distribution (i.i.d.) random variables, each of them takes the values of the constellation set $\{\chi\}_{\chi=0}^{2^{Q}-1}$ and assumed to be equal probable, i.e., $\operatorname{Pr}\left(d_{l}^{(k)}=\chi\right)=1 / 2^{Q}$. Using CCSK modulation, the chip sequence $x_{l}^{(k)}$ of $d_{l}^{(k)}$ is denoted as

$$
\begin{aligned}
x_{l}^{(k)} \triangleq & {\left[\begin{array}{lll}
(k) & x_{l L+1}^{(k)} \cdots & x_{(l+1) L-1}^{(k)}
\end{array}\right]^{T}=\left(\mathbf{M}_{\mathrm{c}}^{(k)}\right)^{T} \mathbf{P}^{d_{l}^{(k)}} \boldsymbol{u} } \\
& \mathbf{M}_{\mathrm{c}}^{(k)} \triangleq \mathbf{C i r c}_{L \times L}\left[m_{0}^{(k)} m_{1}^{(k)} \cdots m_{L-1}^{(k)}\right]
\end{aligned}
$$

where $\mathbf{M}_{c}^{(k)}$ is denoted as $\mathrm{m}$ sequence cyclic shift matrix, $\boldsymbol{m}^{(k)} \triangleq\left[\begin{array}{llll}m_{0}^{(k)} & m_{1}^{(k)} & \cdots & m_{L-1}^{(k)}\end{array}\right]$ is the $\mathrm{m}$ sequence of the $k$-th user, and $\mathbf{P} \triangleq \operatorname{Circ}_{L \times L}\left[\mathbf{0}_{1 \times(L-1)} 1\right] . \quad \boldsymbol{u} \triangleq$ $\left[\begin{array}{ll}1 & \mathbf{0}_{1 \times(L-1)}\end{array}\right]^{T}$, the $i \times j$ matrix $\mathbf{0}_{i \times j}$ contains all zeros, $[\cdot]^{T}$ is the transpose operator. The operator $\operatorname{Circ}_{I \times J}\left[y_{0} y_{1} \cdots y_{J-1}\right]$ applied to a length $J$ vector $(I \leq$ $J)$ returns the cyclic shift matrix, so $\cdot \mathbf{P}^{n} \boldsymbol{u}$ means select the $n$-th Column of a matrix.

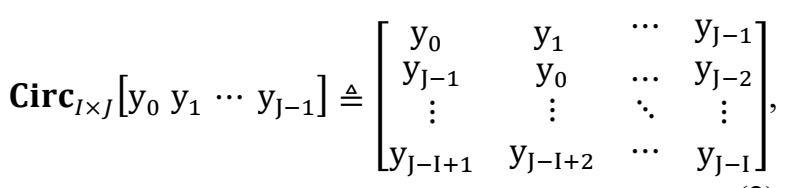

The chip sequence $x_{i}^{(k)}$ feeds a transmitter filter characterized by the impulse response $g(t)$ [11] with

\footnotetext{
* Corresponding author: xiongsi715@163.com
} 
support $\left[0 \mathrm{~T}_{\mathrm{c}}\right]$, so that the transmitted signal of the $k$-th user can be expressed as

$$
s^{(k)}(t)=\sum_{i=-\infty}^{\infty} x_{i}^{(k)} g\left(t-i T_{c}\right)
$$

where $T_{c}$ is denoted as chip duration. The channel finitelength impulse response (FIR) can be expressed as

$$
h^{(k)}(t)=\sum_{n=0}^{N^{(k)}-1} h_{n}^{(k)} \delta\left(t-\tau_{n}^{(k)}\right)
$$

where $\tau_{n}^{(k)}$ is the delay associated with the $n$-th tap, and the set of tap gains $\left\{h_{n}^{(k)}, n=0,1, \ldots, N^{(k)}-1\right.$, $\left.N^{(k)}<L\right\}$ consists of independent complex-valued Gaussian random variables for each user. Thus, the received signal can be expressed as

$$
r(t)=\sum_{k=1}^{K} \sum_{n=0}^{N(k)} \sum_{i=-\infty}^{+\infty} h_{n}^{(k)} x_{i}^{(k)} g\left(t-\tau_{n}^{(k)}-i T_{c}-p^{(k)}\right)
$$

Where $p^{(k)}$ is the arrival time of the $k$-th user, and $w(t)$ is complexadditive white Gaussian noise (AWGN). If ideal timing synchronization is always assumed at the receiver, $r(t)$ is sampled at the epochs $t_{j}^{(l)} \triangleq l L T_{c}+$ $j T_{c}+p^{(k)}$, with $j=0, \cdots, L-1$, which yields a $L$ dimensional vector $\boldsymbol{r}^{(l)} \triangleq\left[r_{0}^{(l)} r_{1}^{(l)} \cdots r_{L-1}^{(l)}\right]^{T}$ with

$$
\begin{gathered}
r_{j}^{(l)}=\sum_{k=1}^{K} \sum_{n=0}^{N^{(k)}-1} h_{n}^{(k)} x_{l L+j-n-\Delta_{p}^{(k)}}^{(k)}+w_{j}^{(l)} \\
\Delta_{p}^{(k)}=<\left(p^{\left(k^{\prime}\right)}-p^{(k)}\right) / T_{c}>, \quad k, k^{\prime}=1 . \cdots, K
\end{gathered}
$$

where $\langle\cdot\rangle$ is the rounding operator. Transmitted over the ISI channel are the last $N^{(k)}$ chips of the block $x_{l-1}^{(k)}$ followed by $x_{l}^{(k)}$ itself. The block $r^{(l)}$ is given as

$$
\begin{gathered}
\boldsymbol{r}^{(l)}=\sum_{k=1}^{K} \mathbf{H}_{c}^{(k)} \widetilde{\mathbf{P}}^{\Delta_{p}^{(k)}} \tilde{\boldsymbol{x}}_{l}^{(k)}+\boldsymbol{w}^{(l)} \\
\mathbf{H}_{c}^{(k)} \triangleq \operatorname{Circ}_{L \times\left(L+N^{(k)}-1\right)}\left[h_{N^{(k)}-1}^{(k)} h_{N^{(k)}-2}^{(k)} \cdots h_{0}^{(k)} \mathbf{0}_{1 \times(L-1)}\right] \\
\widetilde{\mathbf{P}}=\operatorname{Circ}_{\left(L+N^{(k)}-1\right) \times\left(L+N^{(k)}-1\right)}\left[\mathbf{0}_{1 \times\left(L+N^{(k)}-2\right)} 1\right]
\end{gathered}
$$

$\tilde{\boldsymbol{x}}_{l}^{(k)}=\left[\begin{array}{llllll}x_{l L-N}^{(k)}+1 & \cdots & x_{l L-1}^{(k)} & x_{l L}^{(k)} x_{l L+1}^{(k)} & \cdots & x_{(l+1) L-1}^{(k)}\end{array}\right]^{T}(8)$

\section{MAl cancellation using cyclic shift inverse matrix transform}

In the UPPS, the received signals do not have the same power at the receiver end without a closed-loop power control. The strongest user is firstly detected using cyclic cross-correlation. The cyclic cross-correlation may be written in matrix form as follows

$$
\boldsymbol{\rho}^{(\boldsymbol{k})}=\mathbf{M}_{c}^{(k)} \boldsymbol{r}^{(l)}
$$

The cyclic cross-correlation works like the conventional cross-correlation, in which the signal from the $k$-th transmitter is enhanced by the matched-filter gain.
Substituting Eq. (1), Eq. (7) into Eq. (9), and using the property $\mathbf{P}^{i}\left(\mathbf{M}_{c}^{(k)}\right)^{T} \mathbf{P}^{j}=\left(\mathbf{M}_{c}^{(k)}\right)^{T} \mathbf{P}^{i+j}$, Eq. (9) can be written by

$$
\begin{aligned}
& \boldsymbol{\rho}_{l}^{(k)}=\sum_{n=0}^{N^{(k)}} h_{n}^{(k)} \mathbf{M}_{c}^{(k)}\left(\mathbf{M}_{c}^{(k)}\right)^{T} \mathbf{P}^{n+d_{l}^{(k)}} \boldsymbol{u}+\boldsymbol{\varrho} \\
& \boldsymbol{\varrho}=\sum_{k^{\prime}=1, k^{\prime} \neq k}^{K} \sum_{n=0}^{N^{\left(k^{\prime}\right)}} h_{n}^{\left(k^{\prime}\right)} \mathbf{M}_{c}^{(k)}\left(\mathbf{M}_{c}^{\left(k^{\prime}\right)}\right)^{T} \mathbf{P}^{n+\Delta_{p}^{\left(k^{\prime}\right)}+d_{l}^{\left(k^{\prime}\right)}} \boldsymbol{u} \\
& +\sum_{k=1}^{K} \mathbf{M}_{c}^{(k)} \mathbf{H}_{c}^{(k)} \mathbf{P}_{p}^{\Delta_{p}^{(k)}} \Delta_{x_{l}}^{(k)}+\mathbf{M}_{c}^{(k)} \boldsymbol{w}^{(l)} \\
& \Delta_{x_{l}}^{(k)}=\left[x_{l L+1-N^{(k)}}^{(k)}-x_{(l+1) L+1-N^{(k)}}^{(k)} \cdots x_{l L-1}^{(k)}-\right. \\
& \left.\begin{array}{ll}
x_{(l+1) L-1}^{(k)} & \mathbf{0}_{1 \times L}
\end{array}\right]^{T}
\end{aligned}
$$

where $\quad \mathbf{M}_{\mathrm{c}}^{(\mathrm{k})}\left(\mathbf{M}_{\mathrm{c}}^{(\mathrm{k})}\right)^{\mathrm{T}}=\operatorname{Circ}_{\mathrm{L} \times \mathrm{L}}\left[\begin{array}{ll}L & -\mathbf{1}_{1 \times(L-1)}\end{array}\right]$, $\mathbf{M}_{c}^{(k)}\left(\mathbf{M}_{c}^{\left(k^{\prime}\right)}\right)^{T}$ is a cyclic matrix consisting of several fixed values much smaller than $L$. In Eq. (10), the first term contributes to the detection of desired user, the second term $\varrho$ is interference. In Eq. (11), the first term is the MAI that caused by incomplete orthogonal sequences, the second term is the MAI that caused by asynchronous system, the last term is noise interference. The $\hat{d}_{l}^{(k)}$ is

$$
\begin{aligned}
& \hat{d}_{l}^{(k)}=\underset{n=0}{\operatorname{argmax}} \boldsymbol{\rho}_{l}^{(k)} \\
& =\operatorname{argmax}\left(\sum_{n}^{N^{(k)}} h_{n}^{(k)} \mathbf{P}^{n+d_{l}^{(k)}}\left[L,-\mathbf{1}_{1 \times(L-1)}\right]^{T}+\boldsymbol{\varrho}\right)
\end{aligned}
$$

where the $i \times j$ matrix $\mathbf{1}_{i \times j}$ contain all ones. We assumed $\left|h_{0}^{(k)}\right|>\left|h_{n}^{(k)}\right|$, whit $n=1, \cdots, N^{(k)}-1$, and considered far-near effects, the power of the $k$-th user is the strongest, which means $\left|h_{0}^{(k)}\right| \gg\left|h_{n}^{\left(k^{\prime}\right)}\right|, k^{\prime}=$ $1, \cdots, K, k^{\prime} \neq k$. The interference $\varrho$ is negligible, $\hat{d}_{l}^{(k)}=$ $d_{l}^{(k)}$ can be yielded.

From Eq. (13), it can be seen the $\rho_{l}^{(k)}$ will appear some peaks. In order to eliminate the interference of the $k$-th user that has been detected, the signal from the $k$-th transmitter (the interference signal) then may be removed by zeroing out the peaks higher than a certain threshold, $\gamma$. The signals from other users, including the desired signal, which having amplitudes below $\gamma$ and remaining intact [12]. This operation produces the following signal

$$
\begin{aligned}
\widetilde{\boldsymbol{\rho}}_{l}^{(k)} & =\sum_{n=0, \forall n:\left|h_{n}^{(k)}\right|>\gamma}^{N^{(k)}} h_{n}^{(k)} \mathbf{P}^{n+d_{l}^{(k)}}\left[0,-\mathbf{1}_{1 \times L}\right]^{T}+\mathbf{Z} \boldsymbol{\varrho} \\
\mathbf{Z} & =\sum_{n=0, \forall n:\left|h_{n}^{(k)}\right|>\gamma}^{N^{(k)}} \operatorname{diag}\left(\mathbf{P}^{n+d_{l}^{(k)}}\left[\begin{array}{ll}
0 & \mathbf{1}_{1 \times L}
\end{array}\right]^{T}\right)
\end{aligned}
$$


where the operator $\operatorname{diag}(\cdot)$ to be applied to a length $\mathrm{i}$ vector returns a $i \times i$ square matrix with the vector elements along the diagonal. The signal $\tilde{\rho}_{l}^{(\mathrm{k})}$ is now transformed back by using the inverse of the matrix, $\left(\mathrm{M}_{\mathrm{c}}^{(\mathrm{k})}\right)^{-1}$, as follows

$$
\begin{gathered}
\tilde{\boldsymbol{r}}^{(\boldsymbol{l})}=\left(\mathbf{M}_{c}^{(\boldsymbol{k})}\right)^{-\mathbf{1}} \widetilde{\boldsymbol{\rho}}_{\boldsymbol{l}}^{(\boldsymbol{k})}=\dot{\boldsymbol{r}}^{(l)}+(\mathbf{Z}-\mathbf{I}) \dot{\boldsymbol{r}}^{(\boldsymbol{l})}+\dot{\boldsymbol{r}}^{(l)} \\
\dot{\boldsymbol{r}}^{(\boldsymbol{l})}=\sum_{k^{\prime}=1, k^{\prime} \neq k}^{K} \sum_{n=0}^{N^{\left(k^{\prime}\right)}} h_{n}^{\left(k^{\prime}\right)}\left(\mathbf{M}_{c}^{\left(k^{\prime}\right)}\right)^{T} \boldsymbol{P}^{n+\Delta_{p}^{\left(k^{\prime}\right)}+d_{l}^{\left(k^{\prime}\right)} \boldsymbol{u}} \\
\dot{\boldsymbol{r}}^{(l)}=\sum_{n=0, \forall n:\left|h_{n}^{(k)}\right|>\gamma}^{N^{(k)}} h_{n}^{(k)}\left(\mathbf{M}_{c}^{(k)}\right)^{-1} \mathbf{P}^{n+d_{l}^{(k)}} \\
{\left[\mathbf{0 , -}-\mathbf{1}_{1 \times(L-1)}\right]^{\mathbf{T}}+\mathbf{Z} \mathbf{H}_{c}^{(k)} \mathbf{P}_{p}^{\Delta_{p}^{(k)}} \Delta_{x_{l}}^{(k)}}
\end{gathered}
$$

In Eq. (15), I is the identity matrix, the first term is the signal that completely eliminates the $k$-th interfering user, the second term is the interference caused by the zeroing operation, and the third term is the residual interference of the $k$-th user. When the power of the $k$-th interfering user is much greater than the others, the interferences of the 2nd and 3rd terms are almost negligible. The next desired signal can be estimated by matched filtering the data $\tilde{\boldsymbol{r}}^{(l)}$ with the code sequence of the desired source using Eq.(9) again, the above processing is repeated for all users.

\section{$4 \mathrm{MAl}$ cancellation using recursive least square algorithm}

The threshold setting is more sensitive to interference cancellation effects when the power levels of the received user signals are comparable, then the unsuitable threshold settings will reduce the effect of interference cancellation. At this time, we adopt RLS-IC scheme. Before decoding the desired user, the decision information outputted by the upper-level decoder is reconstructed as a reference signal of the MAI, and the RLS algorithm is used for adaptive MAI cancellation [13].

In Fig 2, the receiver of the DS-CDMA system with the RLS-IC process is depicted. The output $\hat{b}_{l}^{(k)}$ of decoder is again encoded, interleaved, and re-spreaded. The regenerated $\hat{x}_{l}^{(k)}$ of detected user is used to estimate the CIRs between the receiver and the detected users by the RLS algorithm [14]. The cost function for obtaining the optimal weight vector of the CIRs is denoted by the weighted least square value of the error, i.e.,

$$
\begin{gathered}
J_{i}^{(k)}=\sum_{j=0}^{i} \lambda^{i-j} e_{i}^{2} \\
e_{i}=r_{i}-\sum_{n=0}^{N^{(k)}-1} \hat{h}_{i-1, n}^{(k)} \hat{x}_{i-n}^{(k)}
\end{gathered}
$$

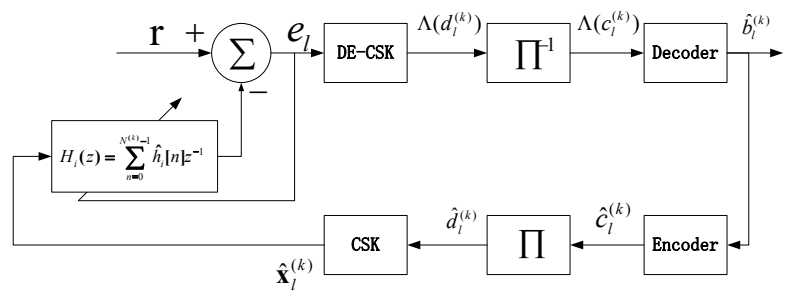

Fig. 2. Block diagram of an adaptive RLS-IC receiver.

where $e_{i}$ is the output of interference cancellation of the $k$-th user, the parameter, $\lambda, 0<\lambda<1$, is a forgetting factor, which controls the speed of convergence and tracking capability of the algorithm. Minimization of $J_{i}^{(k)}$ from the application of the orthogonality principle leads to [13].

$$
\widehat{\boldsymbol{h}}_{i}^{(k)}=\widehat{\boldsymbol{h}}_{i-1}^{(k)}+\mathbf{K}_{i} e_{i}
$$

Where

$$
\begin{gathered}
\widehat{\boldsymbol{h}}_{i}^{(k)}=\left[\begin{array}{lll}
\hat{h}_{i, 0}^{(k)} \hat{h}_{i, 1}^{(k)} & \cdots & \hat{h}_{i, N}^{(k)} \\
\boldsymbol{\mathbf { K }}_{i}=\frac{\boldsymbol{\Sigma}_{i-1} \widehat{\boldsymbol{h}}_{i}^{(k)}}{\lambda^{i}+\left(\widehat{\boldsymbol{h}}_{i}^{(k)}\right)^{H} \boldsymbol{\Sigma}_{i-1}} \\
\boldsymbol{\Sigma}_{i}=\left(\mathbf{1}-\mathbf{K}_{i}\left(\widehat{\boldsymbol{h}}_{i}^{(k)}\right)^{H} \boldsymbol{\Sigma}_{i-1}\right.
\end{array}\right)
\end{gathered}
$$

The interference signal is regenerated based on the detected symbols $\hat{\mathbf{x}}_{i}^{(k)}$ in Fig. 2 and an estimation of the CIRs $\widehat{\boldsymbol{h}}_{i}^{(k)}$ in Eq. (19), and then it's subtracted from the received data to extract the desired signal.

\section{Computer simulations}

A 3-user DS-CDMA system is simulated. 1667 symbols of data are transmitted in total for every user, each symbol is spread with 127 chips, the power of user 1 , user 2 and user 3 are $-20 \mathrm{~dB}, 0 \mathrm{~dB}$ and $-18 \mathrm{~dB}$.

Table 1. Decoding Results of L, J, and D Points.

\begin{tabular}{|c|c|c|c|}
\hline $\begin{array}{c}\text { path } \\
\text { usex }\end{array}$ & $\begin{array}{c}\text { Path 1 } \\
\text { (delay, power ) }\end{array}$ & $\begin{array}{c}\text { Path 2 } \\
\text { (delay, power ) }\end{array}$ & $\begin{array}{c}\text { Path 3 } \\
\text { (delay, power ) }\end{array}$ \\
\hline User 1 & $0 \mathrm{~ms}, 0 \mathrm{~dB}$ & $1 \mathrm{~ms},-13 \mathrm{~dB}$ & $2 \mathrm{~ms},-9 \mathrm{~dB}$ \\
\hline User 2 & $0 \mathrm{~ms}, 0 \mathrm{~dB}$ & $2 \mathrm{~ms},-10 \mathrm{~dB}$ & $5 \mathrm{~ms},-17 \mathrm{~dB}$ \\
\hline User 3 & $0 \mathrm{~ms}, 0 \mathrm{~dB}$ & $3.5 \mathrm{~ms},-6 \mathrm{~dB}$ & $6.3 \mathrm{~ms},-14 \mathrm{~dB}$ \\
\hline
\end{tabular}

Using the arrival time of user 1 as the benchmark, the signals transmitted by user 2 and user 3 arrive late by $1.7 \mathrm{~ms}$ and $3.4 \mathrm{~ms}$ respectively, then each user passes through 3-path Rayleigh Fading Channel, all of multipath delays and powers are shown in Table 1. Assume that user 1 is the expected user, user 2 and user 3 have be detected. Using hybrid IC scheme, the interference of high-power user 2 is firstly mitigated by CSIMT-IC, the interference of user 3 with comparable power is mitigated by RLS-IC. Fig. 3 shows the BERs of the expected user1. It can be seen that the BERs of the hybrid IC scheme are better than the other two algorithms. Because the 2nd and 3rd terms in Eq. (15) 
are not negligible after CSIMT-IC to user 3, the BER performance of CSIMT-IC is the worst.

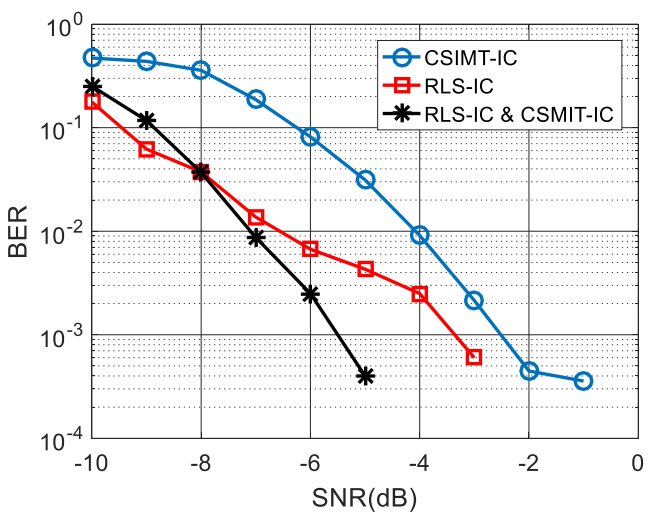

Fig. 3. BERs of the expected user1.

\section{Experimental results}

Considering the experiment complexity, a multi-point transmission and single-point reception communication experiment is designed. When the experiment data is processed offline, the multi-point signal recorded by receiver is directly superposed to simulate multi-user UAC in the UPPS. We assume that there are three positioning base stations at the apex of an equilateral triangle. Each base station synchronously transmits communication signals, and the UUV is passively received.

The experiment was conducted by the Hangzhou Applied Acoustic Research Institution in 2017, which near Dalian Tiger Beach Ocean Park in China. The location of the experiment is shown in Fig.4. The source was submerged from the transmitting ship to a depth of $15 \mathrm{~m}$, and the signals were received at a hydrophone, submerged from near point $\mathrm{O}$ of the wharf to a depth of $10 \mathrm{~m}$, in water depth of about $30 \mathrm{~m}$. The transmitting ships transmitted signals at points L, J, H, G, D and B. The distances from point $\mathrm{O}$ to $\mathrm{L}, \mathrm{J}, \mathrm{H}, \mathrm{G}, \mathrm{D}$ and $\mathrm{B}$ were 2679, 2860, 4993, 5012, 2710 and 647 meters, respectively. At each transmitting point, three-user signals are transmitted, there are 10 groups of each user signal, and 60 multi-user communication results can be obtained through the permutation and combination.

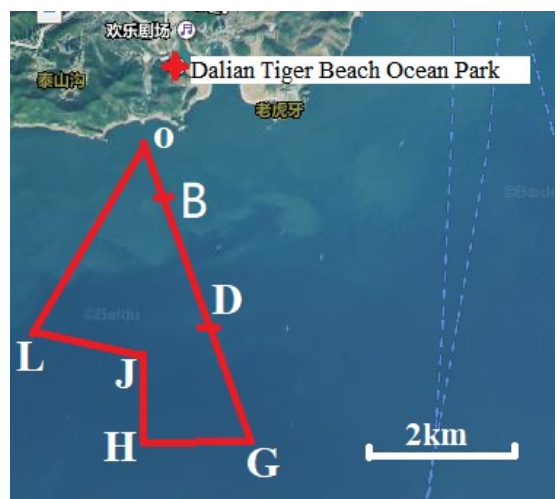

Fig. 4. Experimental position of transmitter and the receiver.

The data block begins with a probe signal (a single m-sequence), followed by the information-bearing signal consisting of 50 symbols, with frequency sweep spanning the bandwidth between $9 \mathrm{kHz}$ and $14 \mathrm{kHz}$, Each symbol is spread with 127 chips, the encoding and decoding of non-binary convolutional codes and the Viterbi algorithms based on soft decision are applied, the data rate is about $100 \mathrm{bps}$. Under the condition of singleuser communication, that is, the received signals were not superposed, the information of three users in all points are decoded correctly. The received signals of the $\mathrm{L}, \mathrm{D}$, and $\mathrm{J}$ points, which are similar to the distance $\mathrm{O}$, that are used for multi-user communication experimental data analysis. Firstly, the synchronization time of the three signals is aligned, and then the signals are directly superposed at different time delays in the time domain to simulate the received asynchronous DS-CDMA signals. Table 1 shows the number of failures of 60 multi-user communications, in which, as long as there is one user decoding error, even a multi-users communication fails. The first column of table 2 is the distance difference, corresponding to different delay of the received signals. It can be seen that because the power of three users is equivalent and there is no far-near effect, the decoding effect of CSIMT-IC is the worst, and the decoding effect of RLS-IC is the best. The received signals at points B, $H$, and $G$ are superposed in the same way. Since the distances of the three points are large, there is a significant far-near effect. For the received signals at points $\mathrm{H}$ and $\mathrm{G}$, the signal at point $\mathrm{B}$ is considered to be strong interference. In Table 3 , it can be seen that the hybrid scheme of RLS-IC and CSIMT-IC has the best decoding effect, when there are high-power users (nearfar effects) and users with comparable power. This situation is very common in the UPPS, and RLS-IC can work better under no far-near effect.

Table 2. Decoding Results of L, J, and D Points.

\begin{tabular}{|c|c|c|c|c|}
\hline $\begin{array}{l}\boldsymbol{d}_{\boldsymbol{L} \boldsymbol{O}} \\
-\boldsymbol{d}_{\boldsymbol{D} \boldsymbol{O}}\end{array}$ & $\begin{array}{c}\text { without } \\
\text { IC }\end{array}$ & $\begin{array}{c}\text { CSIMT- } \\
\text { IC }\end{array}$ & $\begin{array}{c}\text { RLS- } \\
\text { IC }\end{array}$ & $\begin{array}{c}\text { RLS-IC \& } \\
\text { CSIMT-IC }\end{array}$ \\
\hline $0(\mathrm{~m})$ & 16 & 8 & 2 & 6 \\
\hline $50(\mathrm{~m})$ & 21 & 12 & 5 & 9 \\
\hline $100(\mathrm{~m})$ & 25 & 17 & 8 & 11 \\
\hline
\end{tabular}

Table 3. Decoding Results of H, G, and B Points.

\begin{tabular}{|c|c|c|c|c|}
\hline $\begin{array}{l}\boldsymbol{d}_{\boldsymbol{H} \boldsymbol{O}} \\
-\boldsymbol{d}_{\boldsymbol{G O} \boldsymbol{O}}\end{array}$ & $\begin{array}{c}\text { without } \\
\text { IC }\end{array}$ & $\begin{array}{c}\text { CSIMT- } \\
\text { IC }\end{array}$ & $\begin{array}{c}\text { RLS- } \\
\text { IC }\end{array}$ & $\begin{array}{c}\text { RLS-IC \& } \\
\text { CSIMT-IC }\end{array}$ \\
\hline $0(\mathrm{~m})$ & 40 & 9 & 6 & 0 \\
\hline $50(\mathrm{~m})$ & 47 & 8 & 4 & 1 \\
\hline $100(\mathrm{~m})$ & 46 & 13 & 9 & 3 \\
\hline
\end{tabular}

\section{Discussion and summary}

This paper addresses the far-near effect and asynchronism for multi-user communication in the UPPS, where a closed-loop power control is not feasible. We propose a robust hybrid MAI cancellation scheme based on CSIMT and RLS for the DS-CDMA systems using CCSK modulation. The CSIMT-IC is used to suppress the interference which is detected by using cyclic crosscorrelation. The interference signal is removed by hard 
clipping or eliminating the peaks higher than a certain threshold, and projected back the original data space using the inverse matrix, the CSIMT-IC is simple and efficient. The RLS-IC is used to suppress the interference from the detected users with comparable power, the reference signal is reconstructed from the decision information provided by the upper-level decoder, and the RLS algorithm is used for adaptive interference cancellation. The scheme was tested using experimental data. Excellent performance was demonstrated, which owes to the integration of CSIMTIC and RLS-IC that complement both each other. We design the shallow water sea trial near Dalian Tiger Beach Ocean Park in China, The signals are directly superposed at different time delays in the time domain to simulate multi-user UAC in UPPS, using hybrid MAI cancellation, the experimental results show that three users could communicate asynchronously with a failure ratio and match the UPPS application.

This work is partly supported by the National Key Research and Development Program of China (No.2017YFC0305903).

\section{References}

1. N. Leonard, D. Paley, F. Lekian, R. Sepulchre, D. Fratantoni, R. Davis. Proceedings of the IEEE 95, 1 (2007)

2. A. Dareh and A. Boukerche. IEEE Commun. Mag., 53, 11 (2015).

3. L Freitag, $\mathrm{M}$ Grund, $\mathrm{S}$ Singh, et al. IEEE/MTS Oceans Conference (2005).

4. G. Loubet, V. Capellano, and R. Filipiak. IEEE Oceans, 23, 8(1997).

5. S. Tomasin and N. Benvenuto. IEEE Transactions on Wireless Communications, 4, 5 (2005).

6. F. Blackmon, E. Sozer, M. Murandian, J. Proakis, and M. Salehi. Proc. IEEE Oceans, (2001).

7. S. Aliesawi, C. Tsimenidis, B. Sharif, and M. Johnston. Communication Systems Networks and Digital Signal Processing (2010).

8. H. C. Song, J. S. Kim, W. S. Hodgkiss, and W. Kuperman. Journal of the Acoustical Society of America, 128, 5 (2010).

9. S. E. Cho, H. C. Song, and W. S. Hodgkiss. IEEE Journal of Oceanic Engineering, 36, 4 (2011).

10. S. Cho, H. C. Song, and W. Hodgkiss. JASA Express Letters, 131, 2 (2012).

11. F. Pancaldi, and G. M. Vitetta. IEEE Trans. Wireless Commun, vol. 53, 3 (2005).

12. T. C. Yang and Wen-Bin Yang. J. Acoust. 126, 1 (2009).

13. Shiunn-Jang Chern, Chung-Yao Chang. Signal Processing 83, 2 (2003).

14. E. Calvo and M. Stojanovic. IEEE J. Ocean, (2008). 